\title{
DTI mapping of human brain connectivity: statistical fibre tracking and virtual dissection
}

\author{
P. Hagmann, ${ }^{\text {a } b, *}$ J.-P. Thiran, ${ }^{\text {a }}$ L. Jonasson, ${ }^{a}$ P. Vandergheynst, ${ }^{a}$ \\ S. Clarke, ${ }^{\mathrm{c}}$ P. Maeder, ${ }^{\mathrm{b}}$ and R. Meuli ${ }^{\mathrm{b}}$ \\ ${ }^{a}$ Signal Processing Institute, Swiss Federal Institute of Technology, 1015 Lausanne, Switzerland \\ ${ }^{\mathrm{b}}$ Department of Radiology, University Hospital, 1011 Lausanne-CHUV, Switzerland \\ ${ }^{\mathrm{c}}$ Division of Neuropsychology, University Hospital, 1011 Lausanne-CHUV, Switzerland
}

Received 7 October 2002; revised 31 January 2003; accepted 12 February 2003

\begin{abstract}
Several approaches have been used to trace axonal trajectories from diffusion MRI data. If such techniques were first developed in a deterministic framework reducing the diffusion information to one single main direction, more recent approaches emerged that were statistical in nature and that took into account the whole diffusion information. Based on diffusion tensor MRI data coming from normal brains, this paper presents how brain connectivity could be modelled globally by means of a random walk algorithm. The mass of connections thus generated was then virtually dissected to uncover different tracts. Corticospinal, corticobulbar, and corticothalamic tracts, the corpus callosum, the limbic system, several cortical association bundles, the cerebellar peduncles, and the medial lemniscus were all investigated. The results were then displayed in the form of an in vivo brain connectivity atlas. The connectivity pattern and the individual fibre tracts were then compared to known anatomical data; a good matching was found.
\end{abstract}

(C) 2003 Elsevier Science (USA). All rights reserved.

Keywords: Fibre tracking; Diffusion tensor imaging; Connectivity; Brain atlas

\section{Introduction}

Recent developments in diffusion MRI have put this imaging modality to the forefront of interest among the neuroscientific community. The success of diffusion imaging is related to the fact that during their random, diffusiondriven displacements, water molecules probe tissue structure at a microscopic scale well beyond the usual imaging resolution (LeBihan et al., 2001). It has been shown that, in the brain, ordered axonal structure, cell membrane, and myelin sheath strongly influence water diffusion (Beaulieu and Allen, 1994; Beaulieu, 2002) and that there is a direct link between water diffusion and axonal orientation and integrity (Coremans et al., 1994; Wieshmann et al., 1999). In fact, when diffusion tensor (DT) imaging is performed within a compact tract with parallel running axonal trajec-

* Corresponding author. Fax: +41-21-693-76-00.

E-mail address: patric.hagmann@epfl.ch (P. Hagmann). tories like the corticospinal tract, the DT is strongly anisotropic and its principal eigenvector corresponds to the direction of the fibre tract.

These observations were used by several researchers to develop fibre tracking algorithms that all have the same aim: inferring from a DT field the axonal or at least bundles of fibres trajectories - the diameter of an axon being well beyond the resolution of a current MRI scan. Impressive results have been achieved and a wide spectrum of applications is foreseen. A better understanding of diffusion properties in many brain-related diseases, e.g., multiple sclerosis (Maldjian and Grossman, 2001; Filippi et al., 2001), dyslexia (Klingberg et al., 2000), Alzheimer's disease (Rose et al., 2000; Bozzali et al., 2002), schizophrenia (Lim et al., 1999; Foong et al., 2000), brain tumours (Field et al., 2002; Mori et al., 2002a), periventricular leukomalacia (Hoon et al., 2002), as well as spinal cord injury (Mamata et al., 2002) should benefit from those developments. The understanding of normal brain function needs 

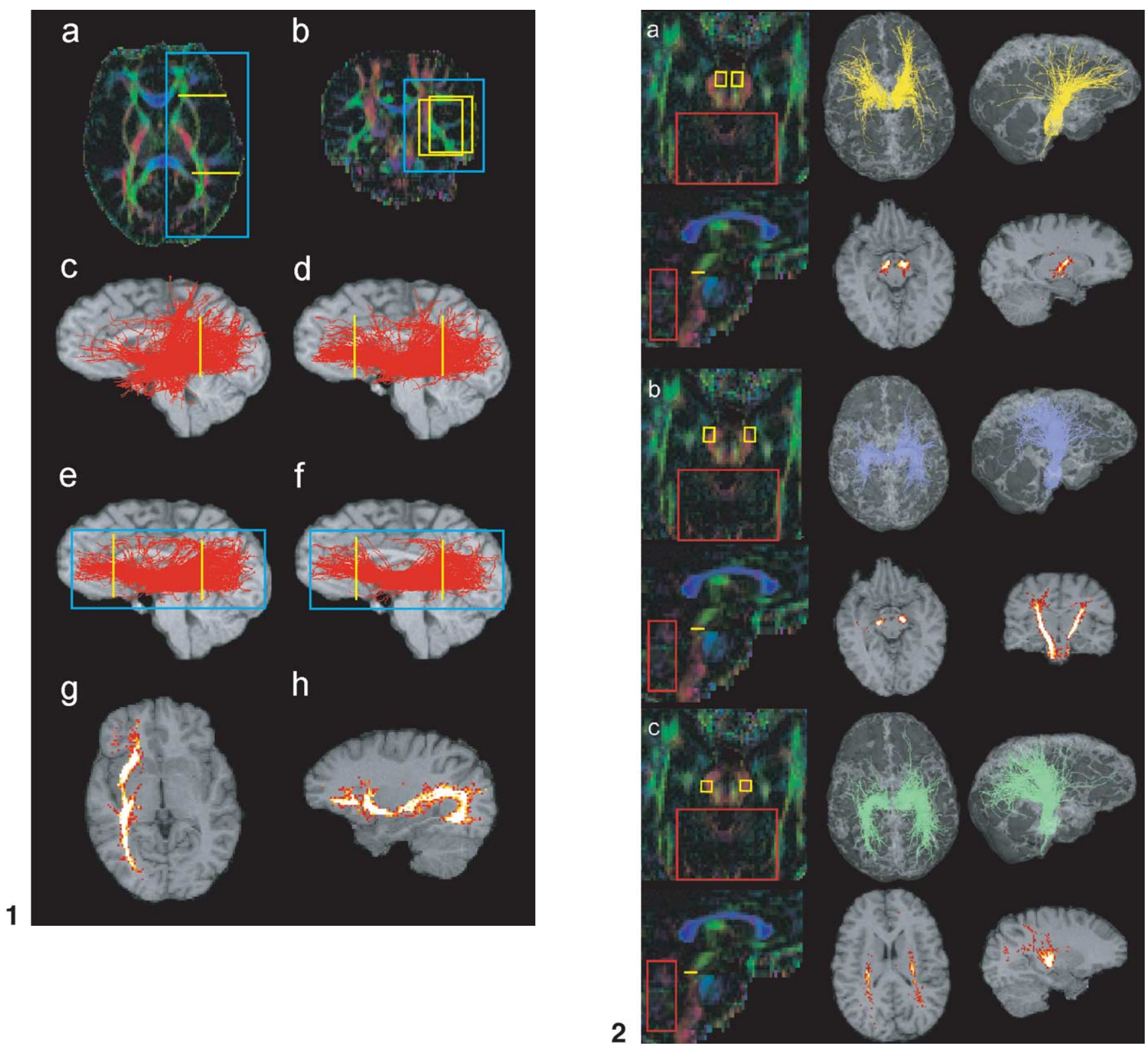

Fig. 1. Virtual dissection of the occipitofrontal fascicle and various connectivity maps as an example of the methodology. (a and b) Colour maps with ROIs. (c) Fibres passing a posteriorly placed yellow ROI. (d) Fibres selected in part c and also passing in the second anterior yellow ROI. (e) Fibres selected in ROI (part d) and that do not pass outside the blue frame. (f) Fibres selected in part e that have a VI above the 20th quantile. (g and h) Statistical density maps of the fibres selected in $\mathrm{f}$.

Fig. 2. Corticobulbar and -spinal tracts. ROIs on colour maps, 3D views, and statistical density maps. (a) Frontopontine tract (yellow), (b) corticospinal tract (blue), (c) parieto-temporo-pontine tract (green).

not only the description of activated cortical areas, like that provided by fMRI, but also a detailed description of the underlying neuronal circuitry.

Most of the algorithms used to infer bundles of fibres from DT imaging are based on a discrete resolution of the integral curves of the vector field corresponding to the reduction of the diffusion tensor to its largest eigenvector (Conturo et al., 1999; Mori et al., 1999; Jones et al., 1999; Basser et al., 2000; Tench et al., 2002). As opposed to those deterministic integral path approaches, this work investi- gates brain circuitry with a statistical random-walk-based algorithm.

\section{Material and methods}

\section{MRI data acquisition}

The images used for this study were acquired with a 1.5 T clinical MRI scanner (Magnetom Symphony; Siemens, Erlangen, Germany). The data were produced with a diffu- 


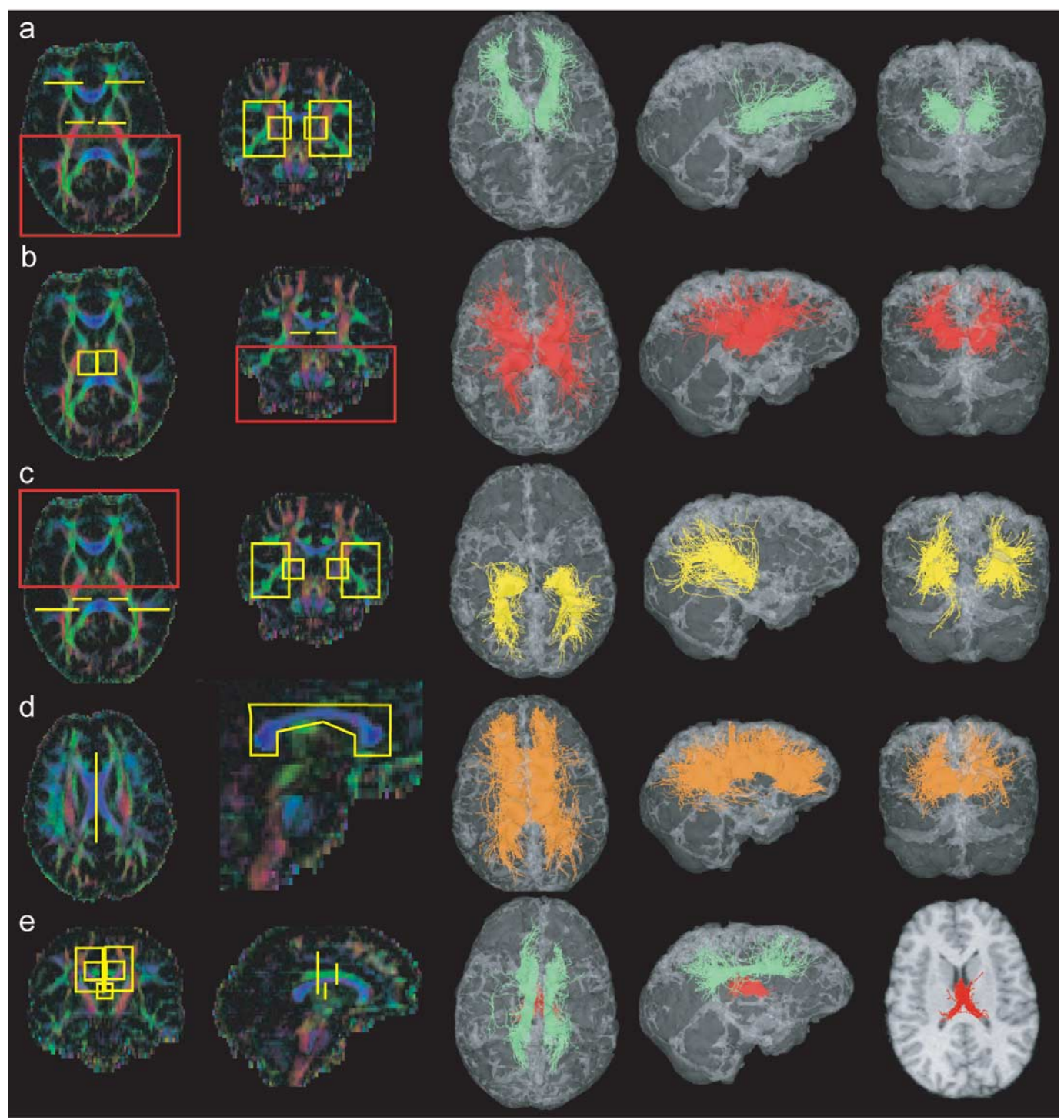

Fig. 3. Corticothalamic, corpus callosum, and limbic system projections. ROIs on colour maps and 3D views. (a) Thalamic frontal projections (green), (b) thalamic superior projections (red), (c) thalamic posterior projections (yellow), (d) corpus callosum projections (orange), (e) cingulum (green) and fornix (red). The lower right image is a projection of the fornix onto an axial slice.

sion-weighted single-shot EPI sequence using the standard Siemens Diffusion Tensor Imaging Package for Symphony. We acquired 44 axial slices in a 128 by 128 matrix size covering the whole brain. The voxel size was 1.64 by 1.64 $\mathrm{mm}$ with a slice thickness of $3.00 \mathrm{~mm}$ without gap. Timing parameters were a TR of $1000 \mathrm{~s}$ and a TE of $89 \mathrm{~s}$. Diffusion weighting was performed along six independent axes according to Basser's polyhedral tessellation (Basser and Pierpaoli, 1998). We used a b-value of $1000 \mathrm{~s} / \mathrm{mm}^{2}$ at a maximum gradient field of $30 \mathrm{mT} / \mathrm{m}$. A normalizing $\mathrm{T}_{2}$ image without diffusion weighting was also acquired. In order to increase the signal-to-noise ratio, the measures were averaged four times. An anatomical $\mathrm{T}_{1}$ magnetization prepared 3D rapid acquisition gradient echo (MPRAGE) was also performed during the same session. The whole examination lasted about $1 \mathrm{~h}$. Images were obtained from two healthy volunteers (one male and one female, both between 25- and 30 -years old). Informed consent from both subjects was obtained in accordance with institutional guidelines.

\section{Data preprocessing}

The data were processed with Matlab 6.1 (The Mathworks, Inc., Natick, MA, USA) running on a standard PC (PIII, $500 \mathrm{MHz}$ ). The DT was computed for each voxel by linear combination of the log-ratio images (Basser and Pierpaoli, 1998). The data were then linearly interpolated in order to obtain a volume with a 3D regular grid of $1.64 \mathrm{~mm}$ by side (matrix of 128 by 128 by 79). We computed then some useful scalar images like the fractional anisotropy (FA) map (Basser and Pierpaoli, 1996) and a colour map (Pajevic and Pierpaoli, 1999). 
An important condition for any fibre tracking algorithm to work properly is to use a good mask on the tensor data. This mask prevents tracking in aberrant areas like the ventricles or outside the brain. It forbids also areas where the tensor data is uncertain for proper tracking of white matter bundles, e.g., the cortex. Most authors used a binary thresholded FA map as stopping criteria, sometimes combined with a coherence measure of the principal eigenvector (Mori et al., 1999; Jones et al., 1999; Basser et al., 2000; Tench et al., 2002). We adopted a similar strategy but used a combination of the normalizing $\mathrm{T}_{2}$-weighted image and the FA map to build our mask. We used the $T_{2}$ water enhancing property to delineate the ventricles. The FA map was median filtered to eliminate the slight salt and pepper effect and get homogeneous white matter regions, and then thresholded at 0.2. Both binary thresholded images were then multiplied in order to achieve precise white matter segmentation. The $\mathrm{T}_{2}$ contribution to the mask was a separation of the ventricles from the white matter and the FA mask segmented the white-grey matter border. Using a relatively low FA threshold and median filtering, the FA map avoided rejection of white matter voxels of low anisotropy due to fibre crossing or noise.

\section{Statistical axonal trajectories}

Water particles animated by thermal heat experience random motion. This Brownian movement can be described by a random walk model. It means that the particle trajectory is made of a succession of jumps that are the realization of a random variable. In an infinite homogeneous medium the distribution of the random variable is an isotropic threedimensional Gaussian function whose variance is proportional to the diffusion coefficient (Einstein, 1956). Living tissue, or more specifically the human brain, is far from a homogeneous medium. It is highly structured and highly compartmented for water particles. Under those circumstances, the diffusion function deviates from an isotropic Gaussian and becomes a complicated function of position and diffusion time (Wedeen et al., 2000). Considering the limitations inherent to a clinical scanner, limited acquisition time and resolution, the DT model is a reasonable compromise to reflect reality. It can be seen as a second order approximation of the average diffusion coefficient over a voxel in any direction of space (Frank, 2002).

In a voxel where all the axons travel in a unique direction, the measured DT exhibits strong anisotropy and the first eigenvector is aligned with the axonal trajectory. But as it was stressed by Pierpaoli et al. (1996) and confirmed by von dem Hagen and Henkelman (2002) experiments, a voxel that contains several populations of axons with different directions has a tensor whose shape will change according to the proportion of each fibre population, moving toward a donutlike or spherical shape. In this situation, the principal eigenvector loses its signification and tracking becomes more hazardous. The principal eigenvector is also very sensitive to noise, especially in areas of low anisotropy (Jones, 2003). The family of tracking algorithms that we could globally describe as deterministic reduce the tensorto a vector-field (Conturo et al., 1999; Mori et al., 1999; Jones et al., 1999; Basser et al., 2000; Tench et al., 2002) and consequently do not take into account the uncertainty of the fibre direction. To address this issue we developed a statistical fibre tracking algorithm based on two hypotheses.

- Considering a voxel, the probability of a fibre to propagate in a given direction is proportional to the corresponding diffusion coefficient. This assumption can be justified by the works of von dem Hagen and Henkelman (2002) showing the positive correlation between the diffusion coefficient and the fibre orientation probability.

- Axonal trajectories or more cautiously trajectories of axonal bundles follow regular curves.

Based on these two ideas we constructed a random walk model of a particle diffusing in a nonhomogeneous medium, here a DT field, $\mathbf{D}^{\alpha}$, with a curve regularizing constraint emphasizing colinearity:

$$
\begin{aligned}
& \mathbf{q}_{i+1}=\mathbf{q}_{i}+\mu \boldsymbol{\Omega}_{i}, \\
& \boldsymbol{\Omega}_{i}=\left\{\begin{array}{c}
\lambda \mathbf{d}_{i}+\boldsymbol{\Omega}_{i-1} \\
\left\|\lambda \mathbf{d}_{i}+\boldsymbol{\Omega}_{i-1}\right\| \\
\boldsymbol{\Omega}_{i} \cdot \boldsymbol{\Omega}_{i-1}>0
\end{array},\right. \\
& \mathbf{d}_{i}=\mathbf{D}_{i}^{\alpha} \mathbf{r}_{i},
\end{aligned}
$$

with $\mathbf{r}_{i}$ a random vector uniformly distributed over a unit sphere. The continuous trajectory of a particle in a 3D Euclidean space is given by its time varying position vector $\mathbf{q}_{i}$, where $i$ is the discrete time step $(0 \leq i \leq m)$. The curve that the particle propagation generates grows along a unit vector $\boldsymbol{\Omega}_{i}$, that is a random direction vector modelling the statistical nature of the diffusion process and the curve regularizing constraint. $\boldsymbol{\Omega}_{i}$ is a weighted sum of the random vector $\mathbf{d}_{i}$, defined on the unit sphere and distributed according to the local diffusive properties ${ }^{1}$ and the previous displacement vector $\boldsymbol{\Omega}_{i-1}$, enhancing colinearity. $\boldsymbol{\Omega}_{i} \cdot \boldsymbol{\Omega}_{i-1}>$ 0 is just an additional constraint to avoid backward jumps. $\mu$ is the step size (here $\mu=0.75$ ), whereas $\alpha$ is an anisotropy enhancing exponent. $\alpha$ is a power to the diffusion matrix $\mathbf{D}$. If $\alpha$ is put to 1 the algorithm gives a lot of weight to possible fibre trajectories deviating from the main direction, whereas if $\alpha$ is large, the distribution is tightened around the main eigenvector in which case the propagation rule comes close to a classic main eigenvector-based fibre tracking. We propose a value of 2 for $\alpha$ as a good compromise between alternative path exploration and near main diffusion direction propagation. $\lambda$ is a constant tuning the relative importance of the random diffusion component vs

\footnotetext{
${ }^{1}$ Here $\mathbf{d}_{i}$ is a single contraction between the nearest neighbour DT, $\mathbf{D}_{i}^{\alpha}$, and a random vector uniformly distributed over a unit sphere, $\mathbf{r}_{i}$.
} 
the curve regularizing term. As $\lambda$ approaches zero, the global regular shape of the curve will be favoured, whereas if set to a large number, more weight is given to the local DT (here $\lambda=1$ ).

In order to map the connectivity of the entire brain, random curves were initiated on a bootstrap of the white matter. This means that a given proportion of the mask voxels, e.g., $40 \%$, were selected randomly to be an initiation point for curves. From those voxels $n$ curves (e.g., 10) were grown in both directions. The curve elongation stopped when a maximum of $m$ steps were performed (e.g., 100, depending of size of the step) or the border of the mask was reached. The result was a statistical estimate of the entire brain connectivity, modelled by a "huge spaghetti plate" of about 100'000 curves. This operation, which needs to be done only once for one DT-MRI dataset, took several hours on our machine with unoptimized Matlab code.

\section{Tract selection or virtual dissection}

In order to visualize anatomical connections in the form of separate identifiable tracts or bundles, a virtual dissection in this modelled brain had to be performed. This was done in two main steps; first, a selection by knowledge-based region of interest (ROI) placement and second, selection by fibre validity classification.

As Mori et al. (2002b) in a deterministic framework, we defined here a ROI as a volume that selects fibres. The manner those ROI were placed needed some attention. Because our approach toward fibre tracking was stochastic, there was a potential risk to map aberrant connections, although those should be very few compared to real tracts. Therefore to avoid a possible bias in the results, we adopted the following general approach:

1. The placement of a first ROI is chosen according to the structure that shall be investigated, guided by the knowledge coming from postmortem anatomical studies.

2. The general structure of the tract appears, though it might be mixed up with other pathways. Their origin, termination, and trajectories can be appreciated.

3. The different tracts selected are identified by confrontation with the postmortem studies.

4. If necessary, a second or a third ROI is selected, in order to separate the tract of interest from the others.

Axonal trajectories were modelled as a result of a stochastic process, thus imbeding in the algorithm the orientational uncertainty related to the DT (Pierpaoli et al., 1996; von dem Hagen and Henkelman, 2002). Among the very large number of curves generated, some accurately match reality whereas others are aberrant. If along an axonal trajectory the tangential diffusion can locally drop due to fibre crossing or fanning in a voxel, we expect the tangential diffusion coefficient averaged along the axonal trajectory to be large.
Therefore, trajectories that follow directions of high diffusion should be more likely than those which do not. To select a posteriori the "good" trajectories, we assigned to each curve a "validity index" (VI), which is the result of an integration of the tangential diffusion coefficient along the trajectory and normalized to the length

$$
\mathrm{VI}=\frac{\sum_{i=1}^{m} \mu \boldsymbol{\Omega}_{i}^{T} \mathbf{D}_{i} \boldsymbol{\Omega}_{i}}{\sum_{i=1}^{m} \mu\left\|\boldsymbol{\Omega}_{i}\right\|}=\frac{1}{m} \sum_{i=1}^{m} \boldsymbol{\Omega}_{i}^{T} \mathbf{D}_{i} \boldsymbol{\Omega}_{i},
$$

where $\boldsymbol{\Omega}_{i}^{T} \mathbf{D}_{i} \boldsymbol{\Omega}_{i}$ is the double contraction of the DT, $\mathbf{D}_{i}$, with the unit displacement vector, $\boldsymbol{\Omega}_{i}$. This contraction is the diffusion coefficient in the displacement direction. For tracts that were selected by one or several ROI, the histogram of the fibre population VI could be plotted. It usually followed a bell-shaped distribution. Fibres below a certain quantile (here $20 \%$ ) were then discarded so that only the most credible fibres with a high VI were retained.

In Fig. 1, we took the example of the occipitofrontal fascicle, an important association bundle (Nieuwenhuys et al., 1988) for illustrating the technique. A first yellow ROI was placed in the caudal part of the brain (Fig. 1c). A large population of fibres was selected of which the occipitofrontal projections as well as fibres of the superior and inferior longitudinal fascicles and fibres belonging to the corona radiata and posterior corticothalamic projections (not visible here in those sagittal figures). Willing to visualise only the occipitofrontal connections, a second yellow ROI was then placed in the frontal brain (Fig. 1d). In some situations, instead of choosing a second ROI, it was more appropriate, because it was less susceptible to bias, to cut out fibres that pass out of a region. We illustrated it here in Fig. 1e with a blue box. The final step of our virtual dissection was to eliminate unlikely fibres that are defined as having a VI inferior to the 20th quantile (Fig. 1f).

We actually used three types of ROI in this paper that have been colour-coded in the following way: (1) yellow region selected fibres that pass through the box, (2) fibres passing in a red box were cut out, and (3) fibres that passed out of a blue box were also discarded.

\section{Various connectivity maps}

The classical way of fibre tract representation is a simple trajectory plot. We also adapted Koch et al. (2002) statistical density maps to our algorithm. From the computed axonal trajectories we summed in each voxel the number of curves that passed through. This measure gave us, for each voxel, a qualitative measure of the probability that a given voxel was connected with the ROI or an empirical measure of the relative amount of fibres of the given tract that passed through that voxel (Fig. 1g and h). We used these two ways 


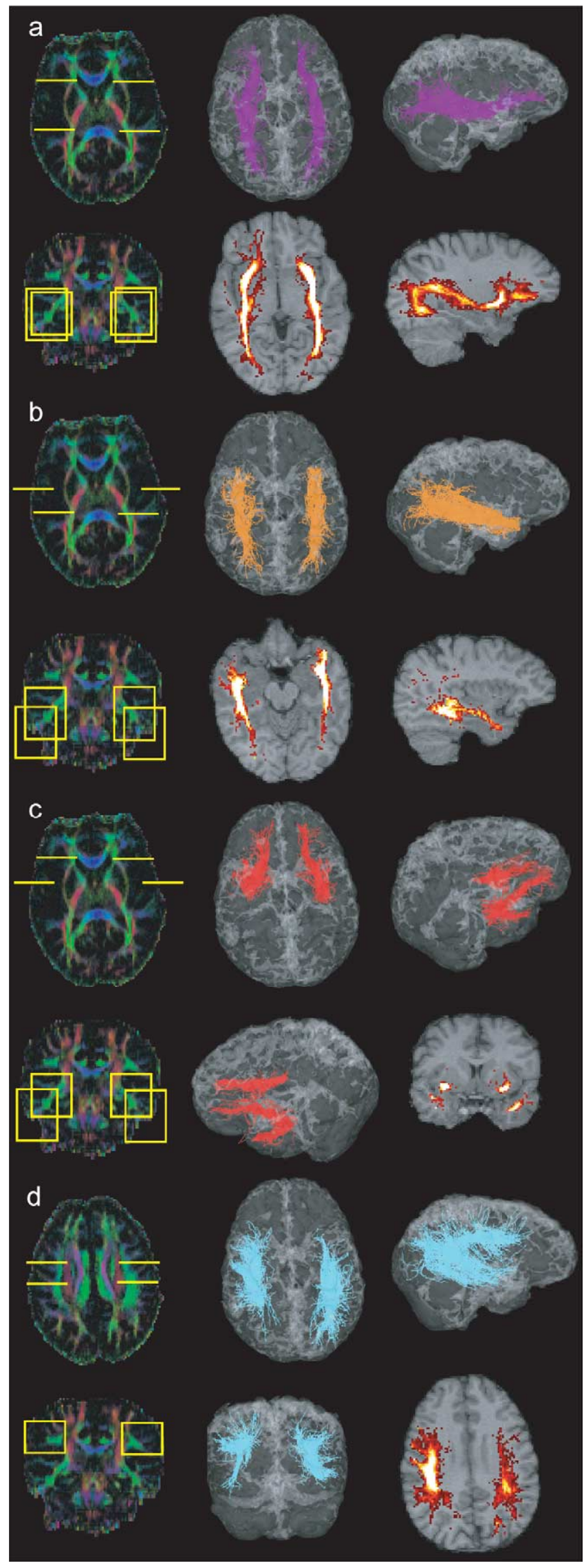

of representing connectivity because they are both useful and complementary.

\section{Results}

The evaluation phase of this research work was performed on two healthy volunteers. It allowed assessing the capacity of our tracking algorithm to infer axonal connectivity by comparing the results to postmortem-based neuroanatomical knowledge, using the Nieuwenhuys et al. (1988) atlas. We applied the above-mentioned methodology in order to perform a virtual dissection of several well-known anatomical systems. This collection of connectivity maps can also be considered to be the beginning of an in vivo brain connectivity atlas. The illustrations are always organized in a similar manner. Two colour maps are used to present the location of the ROI. Some images present the tract of interest on three-dimensional views, whereas a few are projections on an anatomical $\mathrm{T}_{1}$-weighted slice and some others are statistical density maps. We systematically did a bilateral investigation using perfectly symmetric ROI so that side comparison was possible.

\section{Corticobulbar and corticospinal tracts}

The corticospinal tract is a large, well-characterized, and highly anisotropic tract. It has therefore been used by several authors for evaluating their tracking algorithm (Jones et al., 1999; Basser et al., 2000; Poupon et al., 2001; Stieltjes et al., 2001; Koch et al., 2001; Tench et al., 2002; Gossl et al., 2002). The anatomical position of these fibres as well as the position of the corticobulbar tracts is in the brain stem is well described in the literature [for comprehensive summary, see Nieuwenhuys et al. (1988)]. At that level the different bundles separate well. With different locations of the ROIs, we have achieved the dissection of different tracts. For the whole analysis of this subsection, we have excluded fibres coming from the cerebellum on their way to the brain stem.

Fig. 2a shows fibre tract selection by an axial ROI in the ventromedial part of the cerebral peduncles. The fibres thus selected originate mainly in the frontal cortex and are classically described as the frontopontine tract. If the ROI was placed more laterally in the ventrolateral part of the cerebral peduncle, the selected fibres corresponded to the pyramidal tract, originating mainly in the region of the central sulcus and travelling down the brain stem (Fig. 2b). Placing the ROI at the dorsolateral border of the cerebral peduncle

Fig. 4. Cortical association bundles. ROIs on colour maps, 3D views, and statistical density maps. (a) Inferior occipitofrontal fascicle (violet), (b) inferior longitudinal fascicle (orange), (c) uncinate fascicle (red), (d) superior longitudinal fascicle (cyan). 


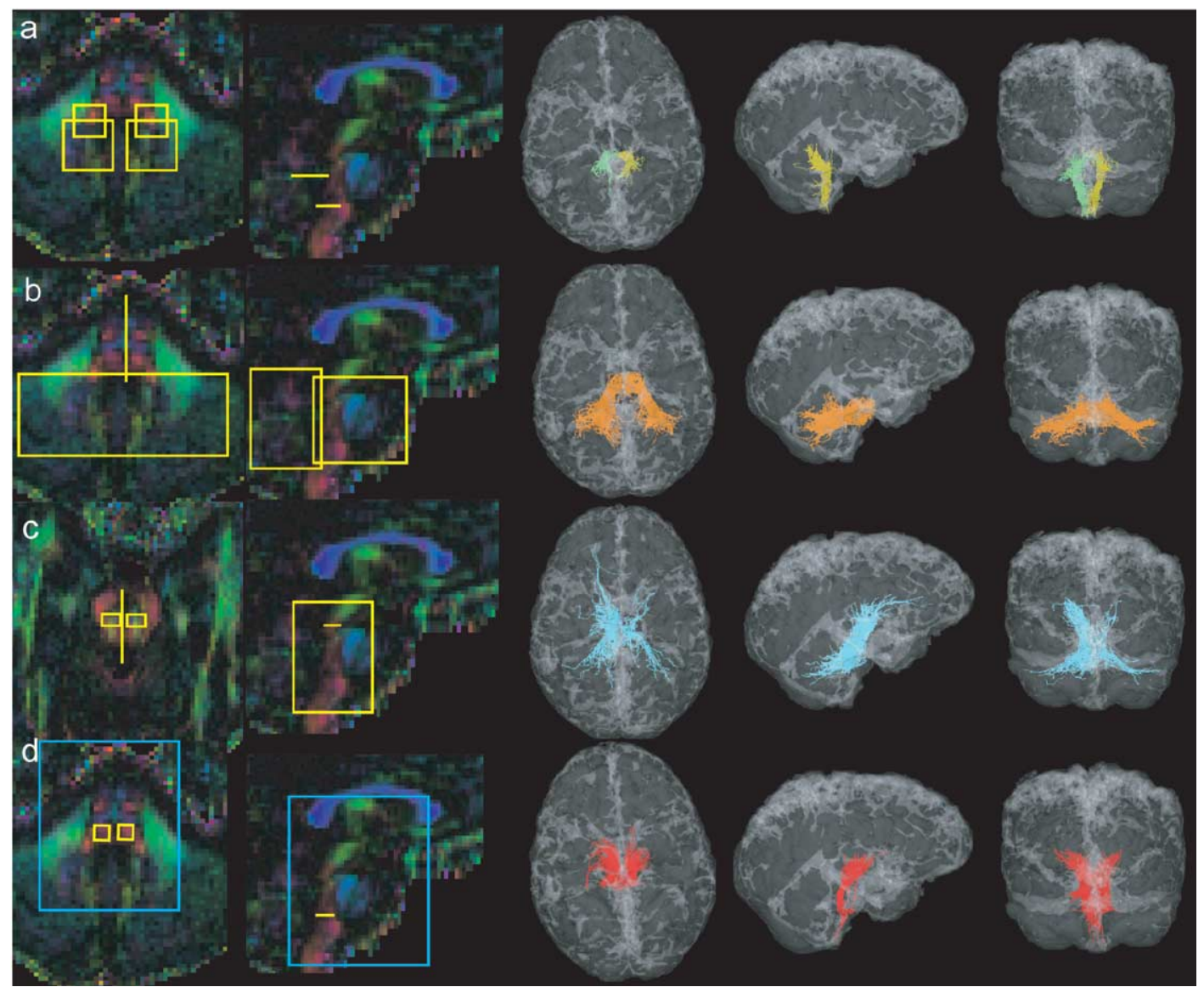

Fig. 5. Cerebellar peduncles and medial lemniscus. ROIs on colour maps, 3D views. (a) Inferior cerebellar peduncle (green and yellow), (b) midcerebellar peduncle (orange), (c) superior cerebellar peduncle (cyan), (d) medial lemniscus (red).

revealed what can be regarded as the parieto-temporo-pontine tract (Fig. 2c).

\section{Corticothalamic projections}

The thalamus sends and receives axons from the different cortical areas. The thalamocortical connections can be roughly separated into three groups, the anterior, superior, and posterior projections.

For selecting the anterior projections, we placed two ROIs in the frontal plane, one in the anterior part of the thalamus, and the other larger ROI in the frontal lobe white matter, anterior to the genu of the corpus callosum (Fig. 3a). Fibres that ran caudally beyond the thalamus were eliminated. In Fig. 3b, we visualised the superior projections by placing an axial ROI in the upper part of the thalamus; fibres running further down were discarded. A similar approach was adopted for the posterior projections by placing ROIs in the frontal plane, one in the posterior part of the thalamus, and another large window in the white matter at the level of the temporooccipital junction (Fig. 3c). Fibres running rostrally beyond the thalamus were eliminated.

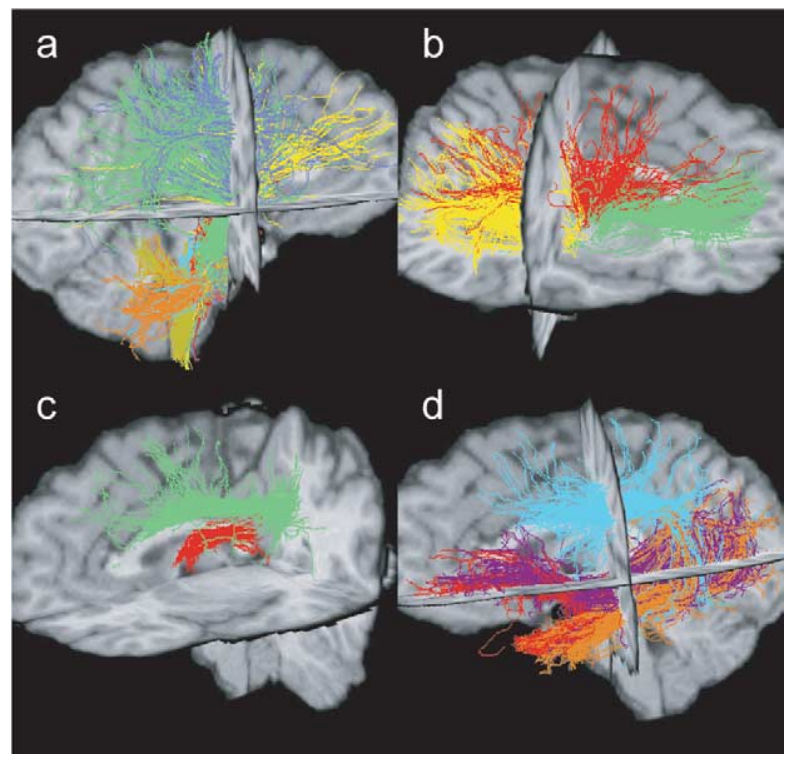

Fig. 6. Three-dimensional view of several systems. (a) Corticobulbar and -spinal tracts, cerebellar peduncles, (b) corticothalamic projections, (c) limbic system, (d) association bundles. 


\section{Corpus callosum and limbic system}

In order to identify fibres passing through the corpus callosum, we placed a large ROI encompassing the whole corpus callosum at the midsagittal plane (Fig. 3d).

Some of the major pathways constituting the limbic system are known to be the fornix and the cingulate bundle. The horizontal portion of the fornix was isolated by placing a ROI in a frontal plane parasagittally beneath the body of the corpus callosum. But both anterior and posterior columns together with the temporal extension could not be identified. This could be due to an insufficient spatial resolution of our DT acquisition technique. The cingulum was identified by using two ROIs placed in a frontal plane, $2 \mathrm{~cm}$ apart within the white matter of the cingulate gyrus (Fig. 3e).

\section{Cortical association bundles}

Corticocortical connections are widespread and form only loose association bundles that are variable in size and shape. Using our method, we visualised four of those major association bundles.

Fig. 4a shows the inferior occipitofrontal fascicle as it was isolated with two ROIs. The first was placed in the posterior parietal and the second in the frontal lobe. In order to isolate the inferior longitudinal fascicle, we also used two selection ROIs. The posterior was, as for the inferior occipitofrontal fascicle, in the posterior parietal lobe, whereas the second in the temporal lobe (Fig. 4b). For identification of the uncinate fascicle, we placed a first ROI in the anterior part of the temporal lobe and a second one in the frontal lobe (Fig. 4c). The superior longitudinal fascicle was selected by two ROI placed below the motor and the posterior parietal cortices in frontal planes (Fig. 4d). This tract shows, interestingly, a clear left-right asymmetry.

\section{Cerebellar peduncles and medial lemniscus}

Here we investigated the relationship between the cerebellum and the brain stem. The three cerebellar peduncles were successively identified by appropriate placement of ROIs. In Fig. 5a, we selected the right and left inferior cerebellar peduncles by means of two ROIs, one in the brain stem and one in the cerebellum. The midcerebellar peduncle was selected by means of one ROI placed across the pons for selecting the fibres that travel in this region laterally and a second ROI was placed on the cerebellum itself (Fig. 5b). The upper cerebellar peduncle was more difficult to isolate and we used the fact that these fibres decussate at the level of the cerebral peduncle to place a ROI in the sagittal plane. Two other ROIs were positioned in an axial plane on each side of the mid line (Fig. 5c). Despite cautious ROI placement, this tract shows an asymmetry in the amount of fibres passing either left or right.

Finally, looking at a colour map at the level of the pons we could easily identify the medial lemniscus that was then selected with another ROI. Those fibres are known to terminate in the thalamus (Fig. 5d), and to visualise this fibre population, we selected the fibres that did not continue beyond it.

\section{Discussion}

We developed a new approach to map brain connectivity that was statistical in nature and based on a global approach toward fibre tracking. This lead to the identification of several fibre tracts (Fig. 6) that all showed accurate correlation with current postmortem-based neuroanatomical knowledge (Nieuwenhuys et al., 1988). Furthermore, previous studies based on other fibre-tracking methods described analogous trajectories for many of those tracts - the pyramidal tract (Jones et al., 1999; Basser et al., 2000; Stieltjes et al., 2001; Poupon et al., 2001; Tench et al., 2002; Gossl et al., 2002), the thalamic projections and the cortical association bundles (Mori et al., 2002b), the brain stem (Stieltjes et al., 2001), and the corpus callosum (Basser et al., 2000; Tench et al., 2002; Poupon et al., 2001—thereby validating our results. The presented data constitute an in vivo brain connectivity atlas.

Both Koch et al. (2002) and our results suggest that statistical fibre tracking is a valuable way for mapping brain connectivity. But it is necessary to notice the clear differences with the classical deterministic fibre-tracking algorithms, and they should be taken into account when interpreting the results. Current deterministic tracking algorithms follow the main eigenvector of the diffusion tensor (Conturo et al., 1999; Mori et al., 1999; Jones et al., 1999; Basser et al., 2000; Tench et al., 2002), reducing the available information. Thus, only the largest and the most homogeneous fibre bundles can be followed, those that do not have a much smaller diameter than the size of a voxel. The identified trajectories are smooth and accurate under the assumption that the signal-to-noise ratio is sufficiently high for limiting the deviation of the tensor field from reality and therefore introducing instability of the discretized differential equation. These problems were investigated by Lazar et al. (2000) as well as by Gossl et al. (2002). Whereas Lazar maps streamlines that are deflected by the local diffusion tensor, Gossl et al. consider fibre tracking in a noisy main eigenvector field as an optimal filtering problem. Gossl's implementation based on a Kalman filter generates curves that follow a corrected main eigenvector in order to minimize the mean square error between the estimated and the underlying ideal tract.

The statistical approach, as presented here, goes one step further. It makes use of the whole diffusion information available in order to explore many potential connections and selects appropriate tract by measuring a posteriori the mean diffusion along the trajectory.

We see here three advantages: 
1. The nature of the data is better taken into account.

2. The sensitivity to noise is decreased.

3. The possibility of fibre crossing and diverging is introduced.

However, an uncertainty concerning the trajectory of individual fibres appears. Each curve should not be interpreted as a precise mapping of a real axonal trajectory.

Selected fibres should be considered together and areas of high fibre density as very likely trajectories whereas projections of few fibres may not correspond to an anatomical entity. In that sense the statistical density mapping seems a complementary tool to the trajectory maps in the context of statistical fibre tracking in order to evaluate the validity of the fibres passing by a given region.

In the presented implementation, several improvements appear also when compared with Koch et al. (2002). Our random walk is continuous as opposed to voxel to voxel jumps. We map individual trajectories and not only statistical density maps. The major drawback of Koch's algorithm which is the dependence of the intensity of the connectivity measure on the distance of the initialization point has been solved in our method by multiple initialization all over the brain.

Our approach, "whole brain simulation" and then virtual dissection, showed striking similarity with known anatomy (Nieuwenhuys et al., 1988). Worth noting are also very obvious left-right asymmetries of the superior longitudinal tract (Fig. 4d) and the upper cerebellar peduncle (Fig. 5c). If inadvertent asymmetric ROI selection and the statistical nature of the algorithm might be a partial explanation, clearly, anatomical difference between both sides is a predominant component, e.g., proportion of grey and white matter (Gur et al., 1980). These observations might be the starting point of asymmetry and male/female dysmorphism study (Clarke et al., 1989).

Using knowledge-based ROI for tract selection is not, at least in the context of statistical fibre tracking, an ideal solution. There remains a risk of biasing the results if the windows are not placed fairly. Therefore, in the future, new solution for ROI placement should be found. Another remaining problem for deterministic as well as statistical fibre tracking is the limited resolution of the imaging scanner and the incapacity of a tensor to model properly multiple fibre tracts in one voxel. High angular resolution imaging (HARDI) (Wedeen et al., 2000; Frank, 2001) seems to be a promising tool for overcoming this limitation.

Finally, fibre tracking is a complex task and different approaches can be valuable. Here, based on a random walk model, we modelled in one shot the global white matter connectivity of healthy volunteers. This model was then virtually dissected in order to identify many different fibre tracts. This collection can be seen as far as we know as a first realization of an in vivo brain connectivity atlas.

\section{Acknowledgments}

We thank Dr. Jean-Marc Vesin (Signal Processing Institute, Swiss Federal Institute of Technology, Lausanne), Eleonora Fornari, and Roberto Martuzzi (Department of Radiology, University Hospital, Lausanne) for their fruitful inputs. Thanks also to Torsten Butz for registering the MR images and to Olivier Cuisenaire for providing the visualization tool (Signal Processing Institute, Swiss Federal Institue of Technology, Lausanne).

\section{References}

Basser, P., Pierpaoli, C., 1996. Microstructural and physiological features of tissues elucidated by quantitative-diffusion-tensor mri. J. Magn. Reson. B 111 (3), 209-219.

Basser, P., Pierpaoli, C., 1998. A simplified method to measure the diffusion tensor from seven mr images. Magn. Reson. Med. 39, 928-934.

Basser, P., Pajevic, S., Pierpaoli, C., Duda, J., Aldroubi, A., 2000. In vivo fiber tractography using dt-mri data. Magn. Reson. Med. 44 (4), 625632.

Beaulieu, C., 2002. Relating micro-structural properties of neural fibres with anisotropic diffusion, in: Proc. Workshop on Diffusion MRI: Biophysical Issues. ISMRM, pp. 79-82.

Beaulieu, C., Allen, P.S., 1994. Determinants of anisotropic water diffusion in nerves. Magn. Reson. Med. 31 (4), 394-400.

Bozzali, M., Falini, A., Franceschi, M., Cercignani, M., Zuffi, M., Scotti, G., Comi, G., Filippi, M., 2002. White matter damage in Alzheimer's disease assessed in vivo using diffusion tensor magnetic resonance imaging. J. Neurol. Neurosurg. Psychiatry 72 (6), 742-746.

Clarke, S., Kraftsik, R., Van der Loos, H., Innocenti, G., 1989. Forms and measures of adult and developing human corpus callosum: is there sexual dimorphism? J. Comp. Neurol. 280 (2), 213-230.

Conturo, T., Lori, N., Cull, T., Akbudak, E., Snyder, A., Shimony, J., McKinstry, R., Burton, H., Raichle, M., 1999. Tracking neuronal fiber pathways in the living human brain. Proc. Natl. Acad. Sci. USA 96 (18), 10422-10427.

Coremans, J., Luypaert, R., Verhelle, F., Stadnik, T., Osteaux, M., 1994. A method for myelin fiber orientation mapping using diffusion-weighted MR images. Magn. Reson. Imaging 12 (3), 443-454.

Einstein, A., 1956. Investigations on the Theory of the Brownian Movement. Dover Publications, New York.

Field, A., Alexander, A., Hasan, K., Arfanakis, K., Witwer, B., Moftakhar, R., Deshmukh, P., Haughton, V., Rowley, H., Noyes, J., B., H., Moritz, C., Meyerand, M., Badie, B., 2002. Diffusion-tensor MR imaging patterns in white matter tracts altered by neoplasm, in: Workshop on Diffusion MRI: Biophysical Issues. ISMRM, pp. 137-140.

Filippi, M., Cercignani, M., Inglese, M., Horsfield, M., Comi, G., 2001. Diffusion tensor magnetic resonance imaging in multiple sclerosis. Neurology 56 (3), 304-311.

Foong, J., Maier, M., Clark, C., Barker, G., Miller, D., Ron, M., 2000. Neuropathological abnormalities of the corpus callosum in schizophrenia: a diffusion tensor imaging study. J. Neurol. Neurosurg. Psychiatry 68 (2), 242-244.

Frank, L., 2001. Anisotropy in high angular resolution diffusion-weighted MRI. Magn. Reson. Med. 45 (6), 935-939.

Frank, L., 2002. Characterization of anisotropy in high angular resolution diffusion-weighted mri. Magn. Reson. Med. 47 (6), 1083-1099.

Gossl, C., Fahrmeir, L., Putz, B., Auer, L., Auer, D., 2002. Fiber tracking from DTI using linear state space models: detectability of the pyramidal tract. Neuroimage 16 (2), 378-388.

Gur, R., Packer, I., Hungerbuhler, J., Reivich, M., Obrist, W., Amarnek, W., Sackeim, H., 1980. Differences in the distribution of gray and 
white matter in human cerebral hemispheres. Science 207 (4436), $1226-1228$.

Hoon, A.J., Lawrie, W.J., Melhem, E., Reinhardt, E., Van Zijl, P., Solaiyappan, M., Jiang, H., Johnston, M., Mori, S., 2002. Diffusion tensor imaging of periventricular leukomalacia shows affected sensory cortex white matter pathways. Neurology 59 (5), 752-756.

Jones, D., 2003. Determining and visualizing uncertainty in estimates of fiber orientation from diffusion tensor mri. Magn. Reson. Med. 49 (1) $7-12$.

Jones, D., Simmons, A., Williams, S., Horsfield, M., 1999. Non-invasive assessment of axonal fiber connectivity in the human brain via diffusion tensor mri. Magn. Reson. Med. 42 (1), 37-41.

Klingberg, T., Hedehus, M., Temple, E., Salz, T., Gabrieli, J., Moseley, M., Poldrack, R., 2000. Microstructure of temporo-parietal white matter as a basis for reading ability: evidence from diffusion tensor magnetic resonance imaging. Neuron 25 (2), 493-500.

Koch, M., Glauche, V., Finsterbusch, J., Nolte, U., Frahm, J., Besuchel, C., 2001. Estimation of anatomical connectivity from diffusion tensor data, in: Proceedings of 7th Annual Meeting of the Organization for Human Brain Mapping, Brighton, HBM, p. 10974.

Koch, M., Norris, D., Hund-Georgiadis, M., 2002. An investigation of functional and anatomical connectivity using magnetic resonance imaging. Neuroimage 16 (1), 241-250.

Lazar, M., Weinstein, D., Hasan, K., Alexander, A., 2000. Axon tractography with tensorlines, in: Proceeding of International Society of Magnetic Resonance in Medicine, Denver, CO, p. 482.

LeBihan, D., Mangin, J., Poupon, C., Clark, C., Pappata, S., Molko, N., Chabriat, H., 2001. Diffusion tensor imaging: concepts and applications. J. Magn. Reson. Imaging 13 (4), 534-546.

Lim, K., Hedehus, M., Moseley, M., de Crespigny, A., Sullivan, E., Pfefferbaum, A., 1999. Compromised white matter tract integrity in schizophrenia inferred from diffusion tensor imaging. Arch. Gen. Psychiatry 56 (4), 367-374.

Maldjian, J., Grossman, R.I., 2001. Future applications of dwi in ms. J. Neurol. Sci. 186(Suppl 1), S55-S57.

Mamata, H., Westin, C., Zou, K., De Girolami, U., F.A., J., S.E., M., 2002. Combined mapping of functional domains and axonal connectivity in cat visual cortex using fmri and dti, in: Workshop on Diffusion MRI: Biophysical Issues, ISMRM, pp. 121-124.

Mori, S., Crain, B., Chacko, V., van Zijl, P., 1999. Three-dimensional tracking of axonal projections in the brain by magnetic resonance imaging. Ann. Neurol. 45 (2), 265-269.
Mori, S., Frederiksen, K., van Zijl, P., Stieltjes, B., Kraut, M., Solaiyappan, M., Pomper, M., 2002a. Brain white matter anatomy of tumor patients evaluated with diffusion tensor imaging. Ann. Neurol. 51 (3), 377-380.

Mori, S., Kaufmann, W., Davatzikos, C., Stieltjes, B., Amodei, L., Fredericksen, K., Pearlson, G., Melhem, E., Solaiyappan, M., Raymond, G., Moser, H., van Zijl, P., 2002b. Imaging cortical association tracts in the human brain using diffusion-tensor-based axonal tracking. Magn. Reson. Med. 47 (2), 215-223.

Nieuwenhuys, R., Voogd, J., van Huijzen, C., 1988. The Human Central Nervous System, 3rd Ed. Springer-Verlag, New York.

Pajevic, S., Pierpaoli, C., 1999. Color schemes to represent the orientation of anisotropic tissues from diffusion tensor data: application to white matter fiber tract mapping in the human brain. Magn. Reson. Med. 42 (3), 526-540

Pierpaoli, C., Jezzard, P., Basser, P., Barnett, A., Di Chiro, G., 1996. Diffusion tensor mr imaging of the human brain. Radiology 201 (3), 637-648

Poupon, C., Mangin, J., Clark, C., Frouin, V., Regis, J., Le Bihan, D., Bloch, I., 2001. Towards inference of human brain connectivity from MR diffusion tensor data. Med. Image Anal. 5 (1), 1-15.

Rose, S., Chen, F., Chalk, J., Zelaya, F., Strugnell, W., Benson, M., Semple, J., Doddrell, D., 2000. Loss of connectivity in alzheimer's disease: an evaluation of white matter tract integrity with colour coded MR diffusion tensor imaging. J. Neurol. Neurosurg. Psychiatry 69 (4), $528-530$.

Stieltjes, B., Kaufmann, W., van Zijl, P., Fredericksen, K., Pearlson, G., Solaiyappan, M., Mori, S., 2001. Diffusion tensor imaging and axonal tracking in the human brainstem. Neuroimage 14 (3), 723-735.

Tench, C., Morgan, P., Wilson, M., Blumhardt, L., 2002. White matter mapping using diffusion tensor MRI. Magn. Reson. Med. 47 (5), 967-972.

von dem Hagen, E., Henkelman, R., 2002. Orientational diffusion reflects fiber structure within a voxel. Magn. Reson. Med. 48 (3), 454-459.

Wedeen, V., Reese, T., Tuch, D., Weigel, M., Dou, J.-G., Weisskoff, R., Chesler, D., 2000. Mapping fiber orentation spectra in cerebral white matter with fourrier-transform diffusion MRI, in: Proceedings of the 8th Annual Meeting of ISMRM, Denver, ISMRM, p. 82.

Wieshmann, U., Clark, C., Symms, M., Franconi, F., Barker, G., Shorvon, S., 1999. Reduced anisotropy of water diffusion in structural cerebral abnormalities demonstrated with diffusion tensor imaging. Magn. Reson. Imaging 17 (9), 1269-1274. 